\title{
Real-time forecasting urban drainage models: full or simplified networks?
}

\section{J. P. Leitão, N. E. Simões, č. Maksimović, F. Ferreira, D. Prodanović, J. S. Matos and A. Sá Marques}

Department of Civil and Environmental Engineering, Imperial College London, Skempton building, South Kensington Campus, London SW7 2AZ, UK E-mail:

joaopaulo.leitao@gmail.com; nuno.simoes08@imperial.ac.uk; c.maksimovic@imperial.ac.uk Department of Civil Engineering and Architecture, IST, Technical University of Lisbon, Av. Rovisco Pais, 1049-001 Lisboa, Portugal E-mail: filipaf@civil.ist.utl.pt; jsm@civil.ist.utl.pt Faculty of Civil Engineering, Institute of Hydraulic Engineering, University of Belgrade, Bulevar Kralja Aleksandra 73, 11000 Belgrade, Serbia E-mail: eprodano@ hikom.grf.bg.ac.rs Department of Civil Engineering, University of Coimbra, Rua Luís Reis Santos, 3030-788 Coimbra, Portugal E-mail: jasm@ dec.uc.pt

\begin{abstract}
Lead time between rainfall prediction results and flood prediction results obtained by hydraulic simulations is one of the crucial factors in the implementation of real-time flood forecasting systems. Therefore, hydraulic simulation times must be as short as possible, with sufficient spatial and temporal flood distribution modelling accuracy. One of the ways to reduce the time required to run hydraulic model simulations is increasing computational speed by simplifying the model networks. This simplification can be conducted by removing and changing some secondary elements using network simplification techniques. The emphasis of this paper is to assess how the level of urban drainage network simplification influences the computational time and overall simulation results' accuracy. The models used in this paper comprise a sewer network and an overland flow drainage system in both $1 \mathrm{D} / 1 \mathrm{D}$ and 1D/2D approaches. The 1D/1D model is used as the reference model to generate several models with different levels of simplifications. The results presented in this paper suggest that the 1D/2D models are not yet suitable to be used in real-time flood prediction applications due to long simulation time, while on the other hand, the simplified 1D/1D models show that considerable reductions in simulation time can be achieved without compromising simulation results (flow and water depth) accuracy.
\end{abstract}

Keywords: flood forecasting; network simplification; urban drainage; 1D/1D and 1D/2D urban drainage models 